\title{
EFFECT OF CHLORIDE ATTACK ON THE PROPERTIES OF CONCRETE PRODUCED BY REPLACING NATURAL SAND BY WASTE FOUNDRY SAND
}

\author{
Sandeep S M ${ }^{1}$, K B Prakash ${ }^{2}$ \\ ${ }^{1}$ P.G Student, Civil Engineering Department, Government Engineering College, Haveri, Karnataka, India \\ ${ }^{2}$ Principal, Government Engineering College, Haveri, Karnataka, India
}

\begin{abstract}
Cement, sand and aggregate are essential desires for any construction industry. Sand is a major material used for preparation of concrete and performs an essential role in mix design. The world wide consumption of natural sand could be very excessive in concrete, due to rapid infrastructure growth. The developing nation like India also facing shortage of good quality natural sand. Rapid extraction of sand from river bed causing serious issues like losing water preserving soil strata, loss of vegetation on the bank of rivers, disturbs the aquatic life as well as disturbs agriculture due to fact that of lowering the water table within the well. Recently natural sand is becoming an expensive material because of its demand within construction industry due to this condition research began for cheap and easily available alternative material to natural sand. Some alternatives materials have already been utilized as a replacement of natural sand such as fly-ash, quarry dust, siliceous stone powder, filtered sand and copper slag are utilized in concrete as a partial or full replacement of natural sand. The main objective of this experimental investigation is to study the effect of replacement of natural sand by foundry sand in various percentages such as $0 \%, 10 \%, 20 \%, 30 \%, 40 \%, 50 \%$, $60 \%, 70 \%$ \& $80 \%$ on the characteristic properties of concrete when subjected to chloride attack. The strength parameters considered are compressive strength, tensile strength, flexural strength, shear strength and impact strength after chloride attack. Chloride content is also found for each replacement after chloride attack.
\end{abstract}

Keywords: Concrete, Natural Sand, Foundary Sand, Cement

\section{INRODUCTION}

Cement, sand and aggregate are essential desires for any construction industry. Sand is a major material used for preparation of concrete and performs an essential role in mix design. The world wide consumption of natural sand could be very excessive in concrete, due to rapid infrastructure growth. The developing nation like India also facing shortage of good quality natural sand. Rapid extraction of sand from river bed causing serious issues like losing water preserving soil strata, loss of vegetation on the bank of rivers, disturbs the aquatic life as well as disturbs agriculture due to fact that of lowering the water table within the well. Recently natural sand is becoming an expensive material because of its demand within construction industry due to this condition research began for cheap and easily available alternative material to natural sand. Some alternatives materials have already been utilized as a replacement of natural sand such as fly-ash, quarry dust, siliceous stone powder, filtered sand and copper slag are utilized in concrete as a partial or full replacement of natural sand.

\subsection{Objective of the Study}

The main objective of this experimental investigation is to study the effect of replacement of natural sand by waste foundry sand in various percentages such as $0 \%, 10 \%, 20 \%$, $30 \%, 40 \%, 50 \%, 60 \%, 70 \%$ and $80 \%$ on the characteristic properties of concrete when subjected to chloride attack.
The strength parameters considered are compressive strength, tensile strength, flexural strength, shear strength and impact strength after chloride attack. Chloride content is also found for each replacement after chloride attack.Chloride content is also found for each replacement after chloride attack.

\subsection{Materials Used}

Cement: Ordinary Portland cementof 43 gradeis used which is conforming to IS: $8112-1989$.

Foundry Sand: Foundry sand used in the experimentation is obtained from the metal industries in Harihar town.

Fine Aggregates: Locally available fine aggregate used in this project conforms to zone II of IS: 383-1970.

Coarse Aggregate: $20 \mathrm{~mm}$ and down size locally available crushed aggregates are used.

Water: Potable water is used for mixing and curing.

\section{METHODOLOGY}

Cement, sand and aggregate were taken in mix proportion 1:1.54:2.65 which correspond to M30 grade of concrete. Fine aggregates were replaced by waste foundry sand in different percentages, such as $0 \%, 10 \%, 20 \%, 30 \%, 40 \%$, 
$50 \%, 60 \%, 70 \%$ and $80 \%$. In the dry mix, required amount of water $(\mathrm{W} / \mathrm{C}=0.45)$ was added and the ingredients of concrete were uniformly mixed in mixer till uniform consistency is achieved. The cubes were casted and compacted on a vibrating table while the prisms were compacted using needle vibrator.The casted specimens to beremoved after 24 hours of casting.

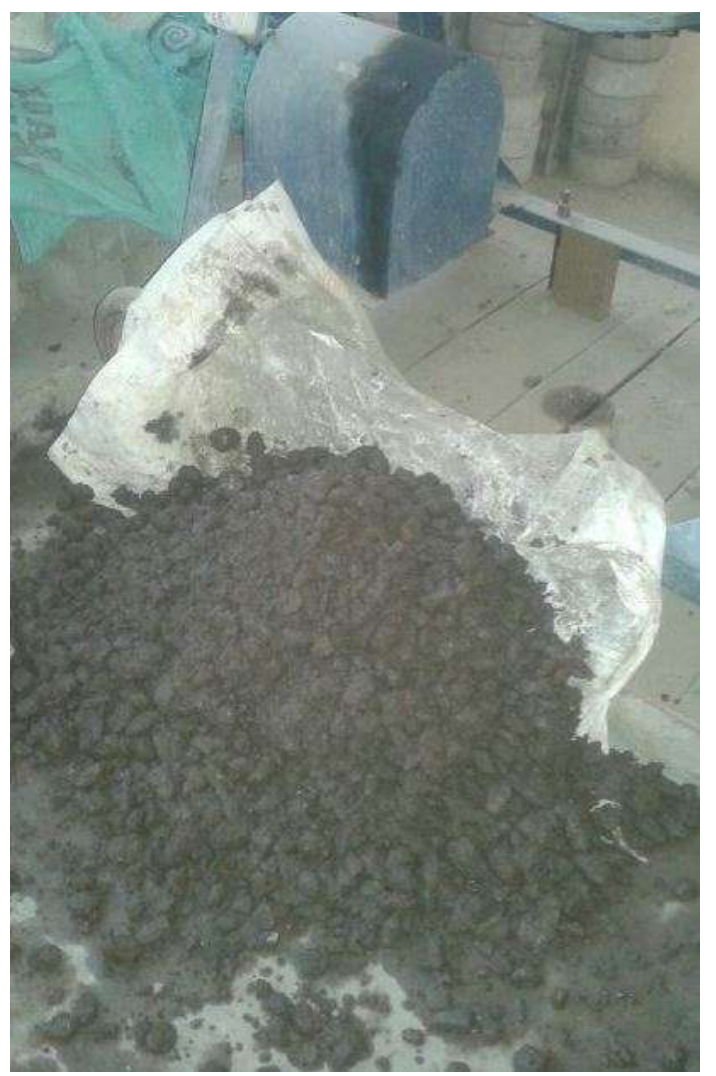

\section{EXPERIMENTAL RESULTS}

Near surface characteristics test results

\subsection{Water Absorption Test Results}

Table 1 gives water absorption test results of concrete for different percentage replacement of fine aggregate bywaste foundry sand with and without chloride attack. The variation in the water absorption is shown in the fig 1.

Table 1: Water absorption test results

\begin{tabular}{|l|l|l|}
\hline Percentage & \multicolumn{2}{|l|}{ Water absorption test results (\%) } \\
\cline { 2 - 3 } $\begin{array}{l}\text { replacement of fine } \\
\text { aggrgate by waste } \\
\text { foundary sand }\end{array}$ & $\begin{array}{l}\text { Withchloride } \\
\text { attack }\end{array}$ & $\begin{array}{l}\text { Without chloride } \\
\text { attack }\end{array}$ \\
\hline 0 & 0.950 & 0.940 \\
\hline 10 & 0.930 & 0.920 \\
\hline 20 & 0.900 & 0.890 \\
\hline 30 & 0.880 & 0.860 \\
\hline 40 & 0.865 & 0.850 \\
\hline $\mathbf{5 0}$ & $\mathbf{0 . 8 5 0}$ & $\mathbf{0 . 8 3 0}$ \\
\hline 60 & 0.870 & 0.855 \\
\hline 70 & 0.880 & 0.865 \\
\hline 80 & 0.900 & 0.880 \\
\hline
\end{tabular}

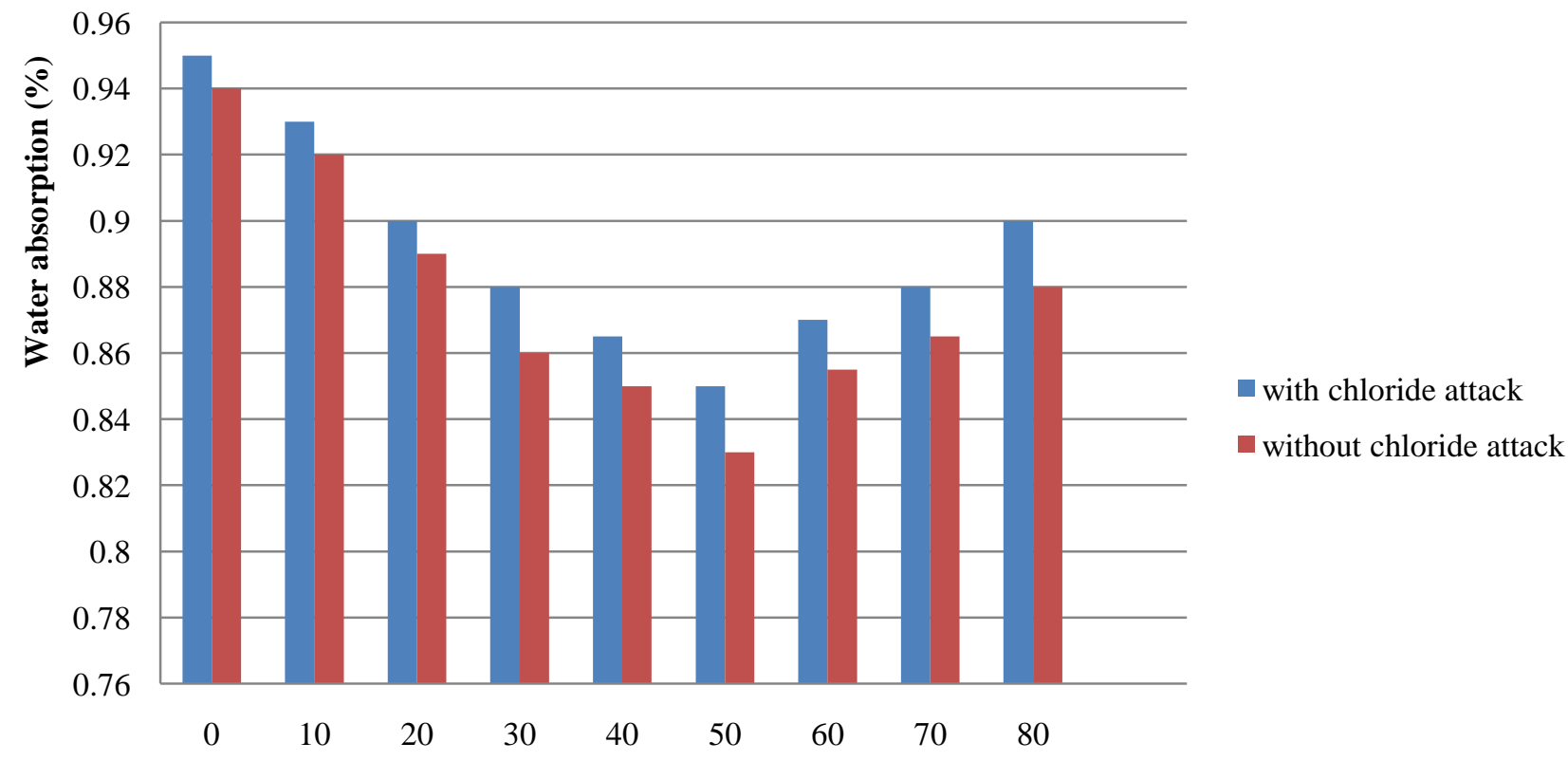

Percentage replacement of fine aggregate by WFS

Fig 1: Variation of water absorption 


\subsection{Sorptivity Test Results}

Table 2 gives sorptivity test results of concrete for different percentage replacement of fine aggregate by foundry sand with and without chloride attack. The variation in sorptivity is shown in fig 2.

Table 2 Sorptivity test result

\begin{tabular}{|c|c|c|}
\hline \multirow{2}{*}{ Percentage replacement of fine aggrgate by waste foundary sand } & \multicolumn{2}{|c|}{ Sorptivity test results $\left(\mathbf{m m} / \mathbf{m i n}^{\mathbf{0 . 5}}\right)$} \\
\cline { 2 - 3 } & With chloride attack & Without chloride attack \\
\hline 0 & 2.96 & 2.92 \\
\hline 10 & 2.90 & 2.85 \\
\hline 20 & 2.84 & 2.80 \\
\hline 30 & 2.75 & 2.71 \\
\hline 40 & 2.68 & 2.60 \\
\hline $\mathbf{5 0}$ & $\mathbf{2 . 5 7}$ & $\mathbf{2 . 5 0}$ \\
\hline 60 & 2.70 & 2.65 \\
\hline 70 & 2.79 & 2.75 \\
\hline 80 & 2.87 & 2.82 \\
\hline
\end{tabular}

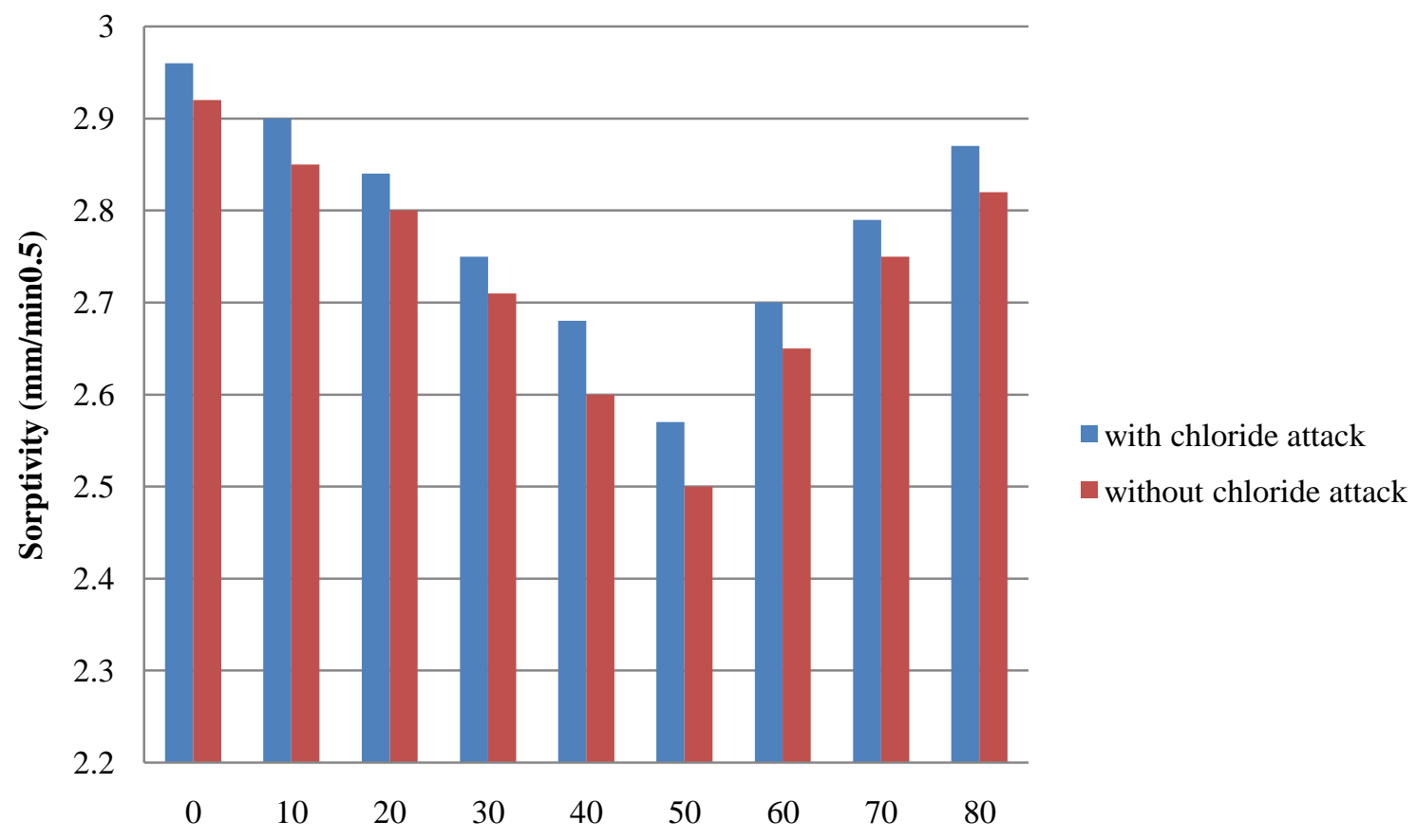

Percentage replacement of fine aggregate by WFS

Fig 2: Variation of sorptivity

\subsection{Strength Test Results}

Compressive strength, tensile strength, flexural strength, shear strength and impact strength test results are given in table 3,4,5, 6 and 7 respectively. The variation of strength is rapicted in the form of graph as shown in fig 3, 4, 5, 6 and 7. 
Table 3: Test results of compressive strength

\begin{tabular}{|l|l|l|l|l|l|}
\hline $\begin{array}{l}\text { Percentage } \\
\text { replacement } \\
\text { of fine } \\
\text { aggregate by } \\
\text { WFS }\end{array}$ & $\begin{array}{l}\text { Compressive } \\
\text { strength of } \\
\text { concrete with } \\
\text { chloride attack } \\
\text { (MPa) }\end{array}$ & $\begin{array}{c}\text { Percentage } \\
\text { increase or } \\
\text { decrease of } \\
\text { compressive } \\
\text { strength with } \\
\text { chloride } \\
\text { attack w.r.t } \\
\text { ref mix }\end{array}$ & $\begin{array}{l}\text { Compressive } \\
\text { strength of } \\
\text { concrete without } \\
\text { chloride attack } \\
\text { (MPa) }\end{array}$ & $\begin{array}{c}\text { Percentage } \\
\text { increase or } \\
\text { decrease of } \\
\text { compressive } \\
\text { strengthwithout } \\
\text { chloride attack } \\
\text { w.r.t } \\
\text { ref mix }\end{array}$ & $\begin{array}{c}\text { Percentage } \\
\text { decrease of } \\
\text { compressive } \\
\text { strength } \\
\text { withchloride } \\
\text { attack }\end{array}$ \\
\hline $0($ Ref $)$ & 30.81 & & 31.10 & & \\
\hline 10 & 31.84 & 3.33 & 32.14 & 3.34 & 0.29 \\
\hline 20 & 32.51 & 5.51 & 33.03 & 6.20 & 11.12 \\
\hline 30 & 33.69 & 9.33 & 34.07 & 9.54 & 2.20 \\
\hline 40 & 34.14 & 10.79 & 35.03 & 12.63 & 14.56 \\
\hline 50 & $\mathbf{3 6 . 5 8}$ & 18.70 & $\mathbf{3 7 . 7 7}$ & 21.44 & 12.77 \\
\hline 60 & 34.44 & 11.77 & 35.33 & 13.60 & 13.45 \\
\hline 70 & 32.29 & 4.79 & 32.88 & 5.72 & 20.90 \\
\hline 80 & 29.47 & 4.34 & 30.14 & 3.08 & \\
\hline
\end{tabular}

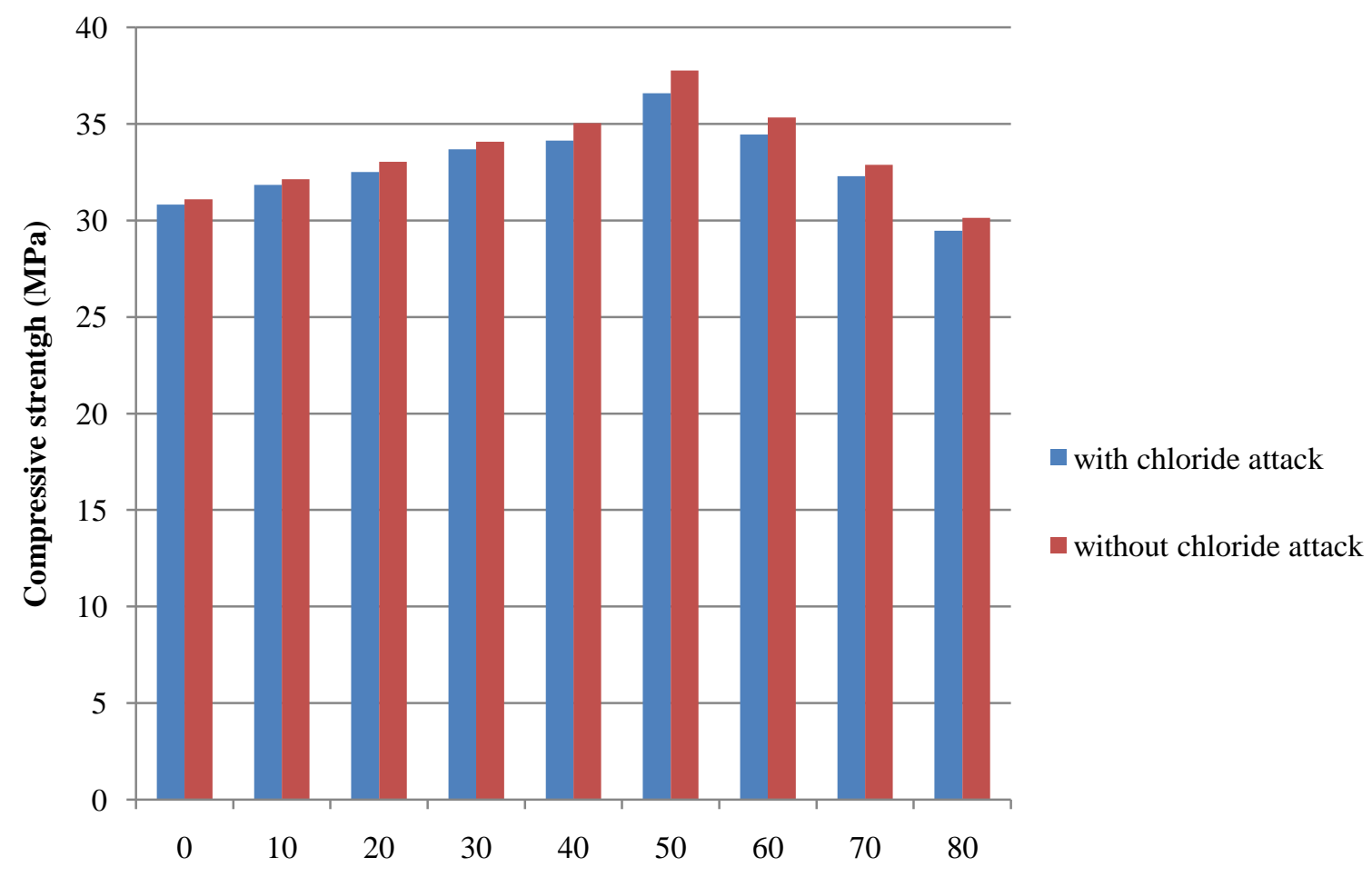

Percentage replacement of fine aggregate by WFS

Fig 3: Variation of compressive strength 
Table 4: Test results of tensile strength

\begin{tabular}{|l|l|l|l|l|l|}
\hline $\begin{array}{l}\text { Percentage } \\
\text { replacement } \\
\text { of fine } \\
\text { aggregate } \\
\text { by WFS }\end{array}$ & $\begin{array}{c}\text { Tensile } \\
\text { strength of } \\
\text { concrete } \\
\text { with chloride } \\
\text { attack } \\
\text { (MPa) }\end{array}$ & $\begin{array}{l}\text { Percentage } \\
\text { increase or } \\
\text { decrease of } \\
\text { tensile } \\
\text { strength with } \\
\text { chloride attack } \\
\text { w.r.t ref mix }\end{array}$ & $\begin{array}{l}\text { Tensile } \\
\text { strength of } \\
\text { concrete } \\
\text { without } \\
\text { chloride attack } \\
\text { (MPa) }\end{array}$ & $\begin{array}{l}\text { Percentage } \\
\text { increase or } \\
\text { decrease of } \\
\text { tensile } \\
\text { strength without } \\
\text { chloride attack } \\
\text { w.r.t ref mix }\end{array}$ & $\begin{array}{l}\text { Percentage } \\
\text { decrease of } \\
\text { tensile } \\
\text { strength } \\
\text { withchloride } \\
\text { attack }\end{array}$ \\
\hline $0($ Ref) & 2.35 & & 2.44 & & \\
\hline 10 & 2.54 & 8.08 & 2.63 & 7.78 & -3.85 \\
\hline 20 & 2.77 & 17.87 & 2.96 & 21.31 & 16.14 \\
\hline 30 & 3.06 & 30.21 & 3.15 & 29.09 & -3.85 \\
\hline 40 & 3.40 & 44.68 & 3.46 & 41.80 & -6.88 \\
\hline 50 & $\mathbf{3 . 5 8}$ & 52.34 & $\mathbf{3 . 6 9}$ & 51.22 & -2.18 \\
\hline 60 & 2.87 & 22.12 & 2.97 & 21.72 & -1.84 \\
\hline 70 & 2.45 & 4.25 & 2.55 & 4.50 & 5.55 \\
\hline 80 & 2.07 & -11.91 & 2.26 & -7.37 & -61.60 \\
\hline
\end{tabular}

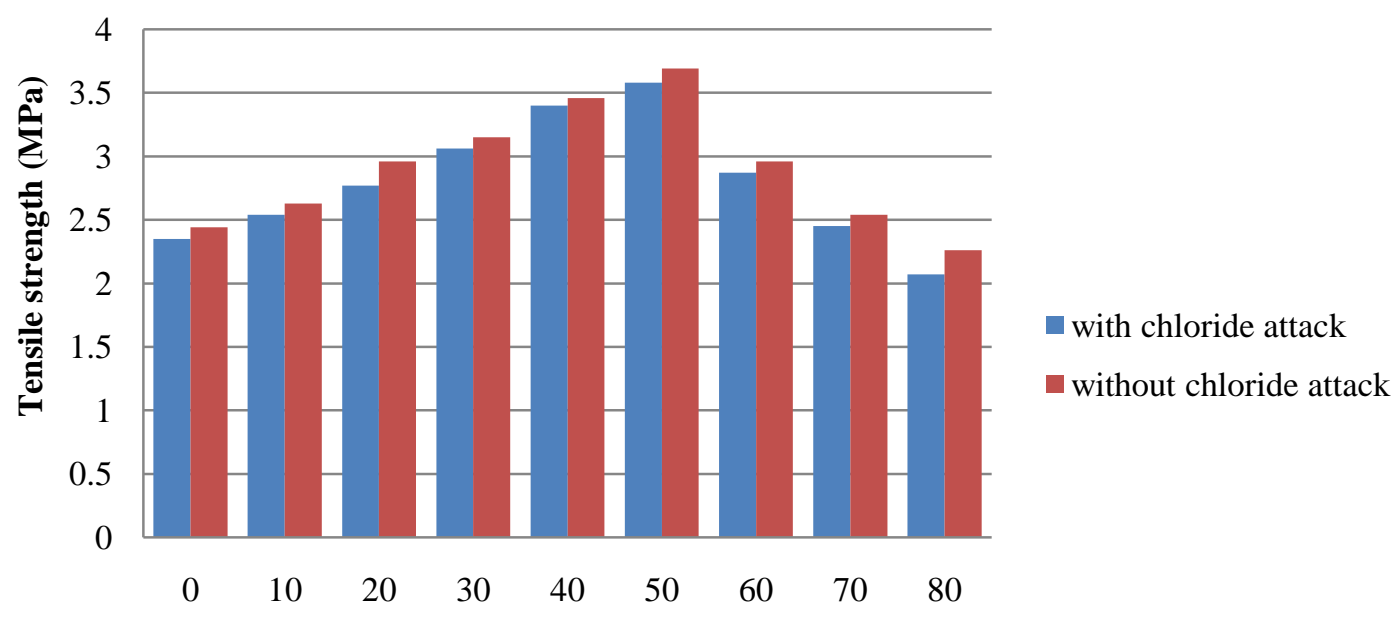

Percentage replacement of fine aggregate by WFS

Fig 4: Variation of tensile strength

Table 5: Test results of flexural strength

\begin{tabular}{|c|c|c|c|c|c|}
\hline $\begin{array}{l}\text { Percentage } \\
\text { replacement } \\
\text { of fine } \\
\text { aggregate } \\
\text { by } \\
\text { WFS }\end{array}$ & $\begin{array}{c}\text { Flexural } \\
\text { strength of } \\
\text { concrete } \\
\text { with chloride } \\
\text { attack (MPa) }\end{array}$ & $\begin{array}{l}\text { Percentage } \\
\text { increase or } \\
\text { decrease of } \\
\text { flexural } \\
\text { strength with } \\
\text { chloride attack } \\
\text { w.r.t ref mix }\end{array}$ & $\begin{array}{l}\text { Flexural } \\
\text { strength of } \\
\text { concrete } \\
\text { without } \\
\text { chloride } \\
\text { attack (MPa) }\end{array}$ & $\begin{array}{l}\text { Percentage } \\
\text { increase or } \\
\text { decrease of } \\
\text { flexural } \\
\text { strength without } \\
\text { chloride attack } \\
\text { w.r.t } \\
\text { ref mix }\end{array}$ & 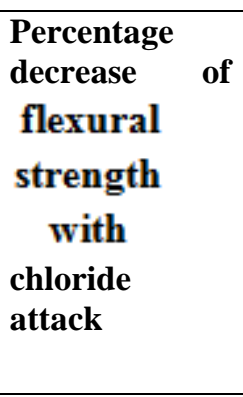 \\
\hline 0 (Ref) & 3.46 & & 3.86 & & \\
\hline 10 & 4.40 & 27.16 & 4.73 & 22.53 & -20.55 \\
\hline 20 & 5.46 & 57.80 & 5.86 & 51.81 & -11.56 \\
\hline 30 & 6.34 & 83.23 & 6.60 & 70.98 & -17.25 \\
\hline 40 & 7.06 & 104.04 & 7.33 & 89.89 & -15.74 \\
\hline 50 & 7.86 & 127.16 & 7.94 & 105.69 & -20.31 \\
\hline 60 & 6.26 & 80.92 & 6.80 & 76.16 & -6.25 \\
\hline 70 & 5.20 & 50.28 & 5.73 & 48.44 & $\begin{array}{l}-3.79 \\
\end{array}$ \\
\hline 80 & 4.53 & 30.92 & 4.67 & 20.98 & -47.37 \\
\hline
\end{tabular}




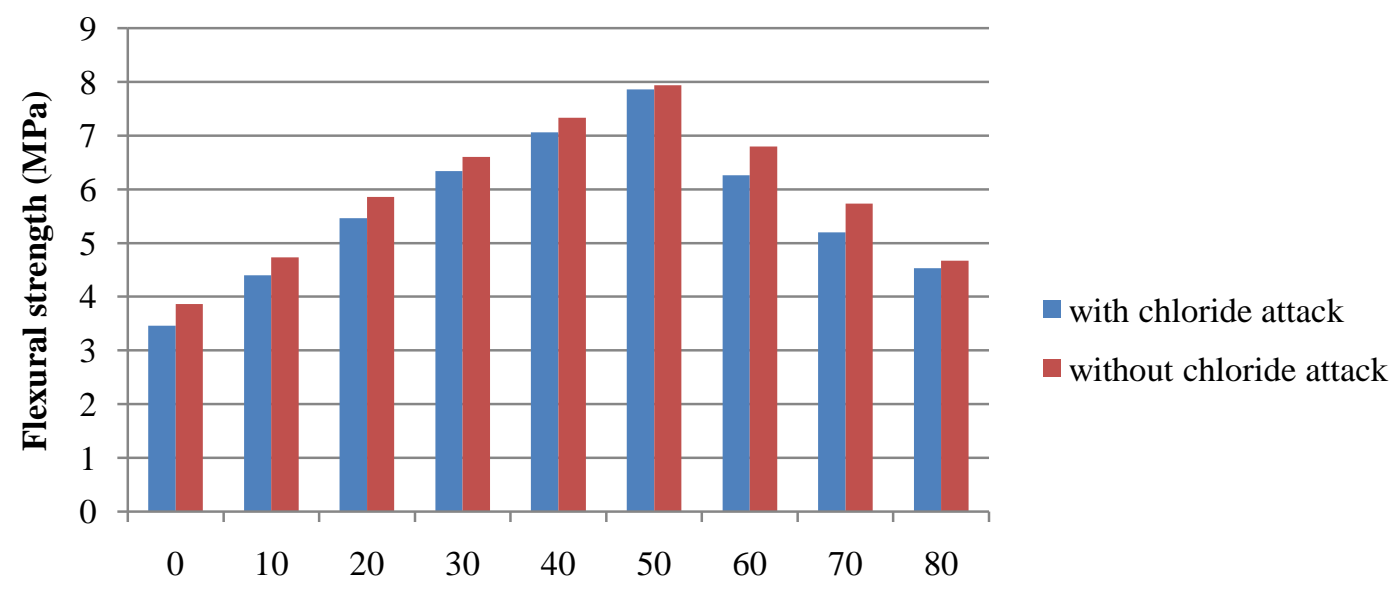

Percentage replacement of fine aggregate by WFS

Fig 5: Variation of flexural strength

Table 6: Test results of shear strength

\begin{tabular}{|c|c|c|c|c|c|}
\hline $\begin{array}{l}\text { Percentage } \\
\text { replacement } \\
\text { of fine } \\
\text { aggregate } \\
\text { byWFS }\end{array}$ & $\begin{array}{l}\text { Shear } \\
\text { strength of } \\
\text { concrete } \\
\text { with chloride } \\
\text { attack } \\
\text { (MPa) }\end{array}$ & $\begin{array}{l}\text { Percentage } \\
\text { increase or } \\
\text { decrease of } \\
\text { shear } \\
\text { strength } \\
\text { with chloride } \\
\text { attack w.r.t ref } \\
\text { mix }\end{array}$ & $\begin{array}{l}\quad \text { Shear } \\
\text { strength of } \\
\quad \text { concrete } \\
\text { without } \\
\text { chloride } \\
\text { attack } \\
\text { (MPa) }\end{array}$ & $\begin{array}{l}\text { Percentage } \\
\text { increase or } \\
\text { decrease of } \\
\text { shear } \\
\text { strength } \\
\text { without } \\
\text { chloride attack } \\
\text { w.r.t ref mix }\end{array}$ & $\begin{array}{c}\text { Percentage } \\
\text { decrease of } \\
\text { shear } \\
\text { strength with } \\
\text { chloride attack }\end{array}$ \\
\hline 0 (Ref) & 1.57 & & 1.85 & & \\
\hline 10 & 2.13 & 35.66 & 2.22 & 20.00 & \begin{tabular}{|l|}
-78.3 \\
\end{tabular} \\
\hline 20 & 2.68 & 70.77 & 2.96 & 60.00 & -17.95 \\
\hline 30 & 3.05 & 94.26 & 3.25 & 75.67 & -24.56 \\
\hline 40 & 3.33 & 112.10 & 3.79 & 104.86 & -6.90 \\
\hline 50 & 3.97 & 152.86 & 4.06 & 119.45 & -27.96 \\
\hline 60 & 3.14 & 100.00 & 3.52 & 90.27 & -10.77 \\
\hline 70 & 2.40 & 52.86 & 2.59 & 40.00 & -32.15 \\
\hline 80 & 1.47 & -6.36 & 1.75 & -5.40 & -17.77 \\
\hline
\end{tabular}

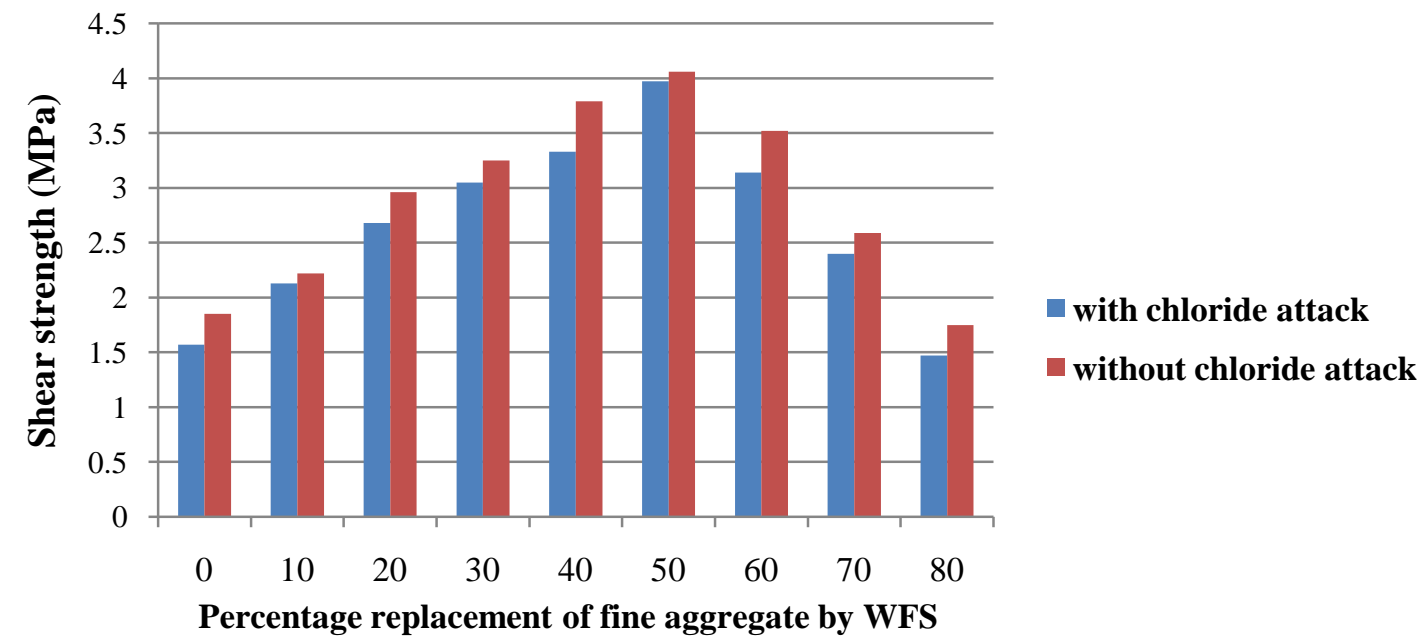

Fig 6: Variation of shear strength 
Table 7: Test results of impact strength

\begin{tabular}{|c|c|c|c|c|c|}
\hline $\begin{array}{l}\text { Percentage } \\
\text { replacement of } \\
\text { fine aggregate } \\
\text { by WFS }\end{array}$ & \begin{tabular}{l}
\multicolumn{1}{c}{ Impact } \\
strength \\
$\quad \quad$ of \\
concrete \\
with chloride \\
attack \\
(N-m)
\end{tabular} & $\begin{array}{l}\text { Percentage } \\
\text { increase or } \\
\text { decrease of } \\
\text { impact } \\
\text { strength with } \\
\text { chloride attack } \\
\text { w.r.t ref mix }\end{array}$ & $\begin{array}{l}\text { Impact } \\
\text { strength } \\
\qquad \quad \text { of } \\
\text { concrete } \\
\text { without } \\
\text { chloride } \\
\text { attack } \\
\text { (N-m) }\end{array}$ & $\begin{array}{l}\text { Percentage } \\
\text { increase or } \\
\text { decrease of } \\
\text { impact } \\
\text { strength without } \\
\text { chloride attack } \\
\text { w.r.t ref mix }\end{array}$ & $\begin{array}{l}\text { Percentage } \\
\text { decrease } \\
\text { of impact } \\
\text { strength } \\
\text { with chloride } \\
\text { attack }\end{array}$ \\
\hline 0 (Ref) & 1065.04 & & 1182.62 & & \\
\hline 10 & 1327.85 & 24.67 & 1417.76 & 19.88 & -24.09 \\
\hline 20 & 1590.66 & 49.35 & 1715.14 & 45.02 & -9.61 \\
\hline 30 & 1832.71 & 72.07 & 1950.28 & 64.91 & -11.03 \\
\hline 40 & 2026.36 & 90.26 & 2220.02 & 87.72 & -2.89 \\
\hline 50 & 2399.82 & 125.32 & 2524.31 & 113.45 & -10.48 \\
\hline 60 & 2012.53 & 88.96 & 2012.44 & 70.16 & -26.79 \\
\hline 70 & 1798.03 & 68.82 & 1977.95 & 67.25 & -2.33 \\
\hline 80 & 1403.93 & 31.81 & 1576.82 & 33.33 & -4.56 \\
\hline
\end{tabular}

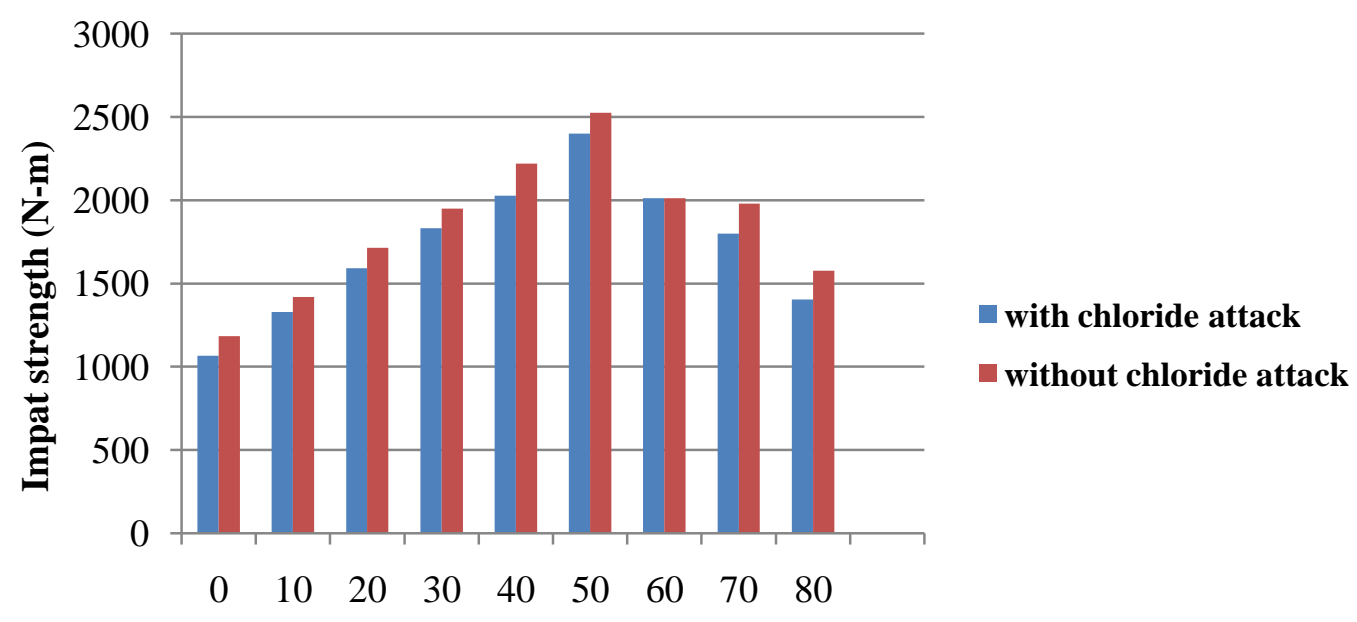

Percentage replacement of fine aggregate by WFS

Fig 7: Variation of impact strength

\subsection{Chloride Test Results}

Table 8 gives the chloride content test results in concrete after chloride attack. Fig. 8 gives the variation of chloride content.

Table 8: Chloride test results

\begin{tabular}{|c|c|c|c|c|c|}
\hline \multirow{2}{*}{\multicolumn{2}{|c|}{$\begin{array}{l}\text { Description of } \\
\text { samples }\end{array}$}} & \multicolumn{3}{|l|}{ Burette reading } & \multirow{2}{*}{$\begin{array}{l}\text { Chloride } \\
\text { Content } \\
\text { (mg/lit) } \\
\end{array}$} \\
\hline & & Initial reading & Final reading & Difference & \\
\hline & & 0.00 & 9.00 & 9.00 & \multirow{2}{*}{134.30} \\
\hline \multicolumn{2}{|l|}{ Distilled water } & 9.00 & 11.30 & 2.30 & \\
\hline \multirow{9}{*}{$\begin{array}{l}\text { Percentage } \\
\text { replacement } \\
\text { of } \\
\text { fine aggregate } \\
\text { by WFS }\end{array}$} & $\mathbf{0}$ & 0.00 & 39.60 & 39.60 & 747.68 \\
\hline & 10 & 0.00 & 36.70 & 36.70 & 689.55 \\
\hline & 20 & 0.00 & 35.50 & 35.50 & 665.49 \\
\hline & 30 & 0.00 & 32.60 & 32.60 & 607.36 \\
\hline & 40 & 0.00 & 30.80 & 30.80 & 571.28 \\
\hline & 50 & 0.00 & 28.90 & 28.90 & 533.20 \\
\hline & 60 & 0.00 & 31.80 & 31.80 & 591.33 \\
\hline & 70 & 0.00 & 33.20 & 33.20 & 619.39 \\
\hline & 80 & 0.00 & 34.60 & 34.60 & 647.45 \\
\hline
\end{tabular}




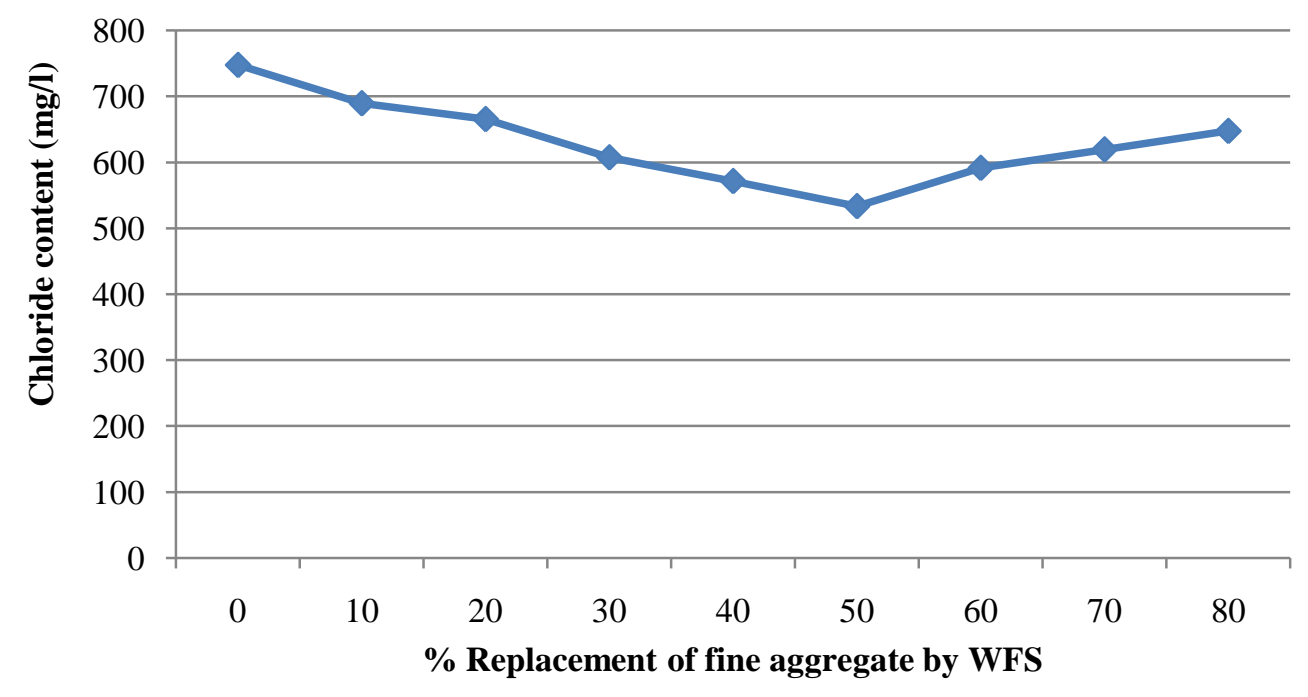

Fig 8: Variation of chloride content

\section{CONCLUSION}

Following conclusions may be drawn on the study conducted on the behavior of concrete containing waste foundry sand under chloride attack.

$>$ Minimum values of water absorption and sorptivity may be obtained by replacing $50 \%$ fine aggregate by waste foundry sand. It is true for concrete with chloride attack and without chloride attack.

$>$ Water absorption and sorptivityare little higher for the concrete produced by replacing fine aggregate by waste foundry sand, when subjected to chloride attack.

$>$ Higher values of strength characteristics for concrete such as compressive strength, tensile strength, flexural strength, shear strength and impact strength may be obtained by replacing $50 \%$ replacement of fine aggregate by waste foundry sand. This is true for concrete with chloride attack and without chloride attack.

$>$ Strength characteristics for concrete produced by replacement of fine aggregate by waste foundry sand and without subjecting to chloride attack exhibit higher values as compared to the concrete which is subjected to chloride attack.

$>50 \%$ replacement of fine aggregate by waste foundry sand yields low chloride content.

\section{REFERENCES}

[1] Smit M Kacha, Abhay V. NakumAnkur C. Bhogayata, "Use of used foundry sand in concrete: A state of art review" International Journal of Research in Engineering and Technology (IJRET) E-ISSN: 23191163, PISSN: 2321-7308 Volume: 03 Issue: 02 Feb2014.

[2] Abdul Wahab,Sohail M D, "A study on the mechanical properties of concrete by replacing sand with waste foundry sand" International Journal of Engineering Technology and Advanced Engineering (IJETAE) ISSN: 2250-2459 Vol. 3, Issue 11, November 2013.
[3] Pradeep Kumar D, Eswarmoorthi P, "Strength characteristics of structural concrete elements using foundry sand" International Journal of Engineering Research and Applications (IJERA) ISSN: 2248-9622 trends and recent advances in Civil Engineering (TRACE-24 $4^{\text {th }}-25^{\text {th }}$ January 2014).

[4] Eknath P Salokhe,Desai D B, "Application of foundry waste sand in manufacture of concrete" IOSR Journal of Mechanical and Civil Engineering (Iosr-Jmce) ISSN: 2278-1684, PP: 43-48.

[5] Dushyant R Bhimani, Jayesh Kumar Pitroda, Jaydev J. Bhavsar, "Effect of used foundry sand and pozzocrete partial replacement with fine aggregate and cement in concrete" International Journal of Innovative Technology and Exploring Engineering (IJITEE) ISSN: 2278-3075, Volume-2, Issue-4, March 2013.

[6] SaveriaMonosi, Daniela Sani And Francesca Tittarelli, "Used foundry sand in cement mortars and concrete production" The Open Waste Management Journal, 2010, vol 03, PP 18-25.

[7] Vipul D. Prajapati, Nilay Joshi, Jayeshkumar Pitroda, "Techno-economical study of rigid pavement by using the used foundry sand". International Journal of Engineering Inventions e-ISSN:2278-7461, p-ISSN: 2319-6491 Volume 4, issue7 (December 2014).

[8] Khuram Rashid, Muhammad AkramTahir, Sana Nazir, "Evaluation of concrete compressive strength by incorporating used foundry sand" American Journal of Engineering Research (AJER) e-ISSN: 2320-0847, pISSN: 2320-0936 Volume: 03, Issue:02, 2014.

[9] Pathariya Saraswati C , Rana Jaykrishna K , Patel Ankit N, "Application of waste foundry sand for evolution of low-cost concrete" International Journal of Engineering Trends and Technology (IJETT)- Volume 4, Issue 10, 0ct-2013.

[10] IS: 456-2000, "Plain and Reinforced Concrete-Code Practice" (Fourth Revision), Bureau of Indian Standards, ManakBhavan, \#9,Bahadur Shah ZafarMarg, New Delhi, October 2000. 
[11] IS: 8112-1989, "43 Grade Ordinary Portland CementSpecifications" (First Revision), Bureau of Indian Standards, ManakBhavan, \#9,Bahadur Shah ZafarMarg, New Delhi, May 1990.

[12] IS: 383-1970, "Specifications For Coarse And Fine Aggregate From Natural Sources For Concrete" (Second Revision), Bureau Of Indian Standards, ManakBhavan, 9 Bahadur Shah ZafarMarg, New Delhi, April 1971.

[13] IS: 516 1959, "Methods Of Tests For Strength Of Concrete", Edition 1.2, BureauOfindian Standards, Manak Bhavan, No9, Bahadur Shah Zafar Marg, New Delhi, Reaffirmed 1999.

[14] Santhakumar A. R. "Concrete Technology", Seventh Impression, Oxford University Press, YMCA Library Building, Jai Singh Road, New Delhi-110 001, ISBN13: 978-0-19-567153-7, 2011.

[15] Shetty MS., "Concrete Technology,Theory And Practice" Sixth (Multicolour Illustrative) Edition, S. Chand \& Company Ltd. (An ISO 9001:2000 Company), Ram Nagar, New Delhi-110 055,ISBN:81219-0003-4, Reprint 2009. 